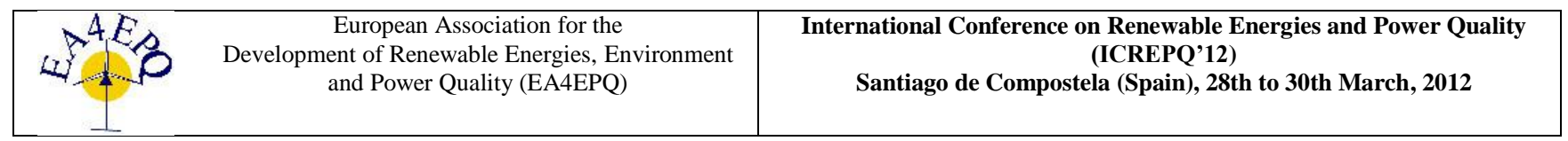

\title{
Stability Support of Autonomous Networks with Dispersed Generation Using Rotary Ride Through Systems
}

\author{
ir. J.C. Soriano ${ }^{1}$, ir. P. Karaliolios ${ }^{1}$, ir. E. Beermann ${ }^{2}$, Prof. dr.ir. J.F.G. Cobben ${ }^{1}$, Prof.ir. W.L. Kling ${ }^{1}$ \\ ${ }^{1}$ Department of Electrical Engineering \\ Electrical Energy Systems, University of Technology Eindhoven \\ Eindhoven, 5600 MB, P.O. Box 513, The Netherlands \\ Phone/Fax number: +31(0)40-247 9111, 31(0)40-245 6087, e-mail: j.c.soriano.montero@alumnus.tue.nl, \\ p.karaliolios@.tue.nl, J.F.G.Cobben@tue.nl, w.l.kling@tue.nl, \\ 2 HITEC Power Protection \\ Bedrijvenpark Twente no. 40 \\ 7602 KB Almelo, The Netherlands \\ Phone/fax: Phone: +31 546589 589, +31 546589 489, e-mail: e.beermann@ hitec-ups.com
}

\begin{abstract}
Dispersed generation (DG) could allow networks to operate autonomously. However, technical challenges need to be addressed to diminish transients in voltage and frequency. In this paper a commercial flywheel ride through system (FRT) is modeled using DigSILENT Power Factory and validated using experimental data. The model is tested with dispersed generation in a test network to simulate transients when disconnection of the grid occurs. The model is also simulated in two study cases: a remote autonomous network supplied by a wind farm and a section of the Dutch electricity grid called "Tiel Zuid" with high penetration of dispersed generation. The results indicate that a FRT can successfully be used to stabilize frequency within regulation limits and maintain stable conditions during disconnections. Additionally, the number of dispersed generation units and the voltage control deeply affect the performance of the RTs and the behavior of the grid.
\end{abstract}

\section{Key Words}

Flywheels, UPS, autonomous networks, dispersed generation, Microgrids.

\section{Introduction}

The rising implementation of DG, apart from its challenges, gives the possibility to export energy back to the grid. With high penetration of DG, it is possible to encapsulate areas and control their power flow allowing certain regions to work autonomously from the network and built Microgrids. This brings new challenges to maintain stable voltage levels, frequency and load behavior [1]. Furthermore, the successful operation of autonomous networks and Microgrids rely on storage systems to balance power and energy demand, and to have seamless transition between grid-connected and autonomous mode. These storage systems include batteries, capacitors, superconducting magnetic energy storage (SMES) and flywheels [2], each with its characteristic power and energy densities. FRT systems can be used to support grid disconnection, variability of renewable energy sources and to facilitate stable autonomous networks. In this paper, a commercial rotary RT system from the company HITEC Power Protection is modeled using the software DigSILENT Power Factory. The model is tested in networks that include DG units such as combined heat and power systems (CHPs) and wind turbines. Furthermore, the model is tested in two study cases; a remote autonomous network and a section of the Dutch electricity grid named "Tiel Zuid" which has a high penetration level of CHP.

\section{Background}

DG is production of electricity in the medium or low voltage network ranges. Energy is transferred in a bidirectional which brings new challenges to maintain quality of power delivery, especially with high penetration of renewable energy technologies. DG has some benefits [3]: Improves reliability of the network, reduces losses, defers future investments, reduces carbon emissions, allows autonomous operation, reduces energy demand, and assists in the creation of Microgrids.

Flywheels store energy in the form of rotational energy. Flywheels are usually placed in vacuum to eliminate the friction from the air. The amount of energy that can be stored in the flywheel is a function of the rotating mass, shape and speed (Eq.1).

$$
E_{k}=\frac{1}{2} \boldsymbol{I} \omega^{2}
$$

The speed, size and application of flywheels vary from the automotive industry to uninterruptible power supply systems (UPSs).

\section{Rotary Ride Through System Model}

The HITEC FRT consists of a flywheel, an induction coupling and a generator (Fig.1). The stored kinetic energy is transferred from the inner rotor to the outer rotor driving 
the alternator. The alternator will remain at constant speed of $1500 \mathrm{rpm}$ while the flywheel speed decreases (starting at $2900 \mathrm{rpm}$ ). The output power of the system will be triggered by the frequency drop at the node, giving enough torque to the rotor and support the load.

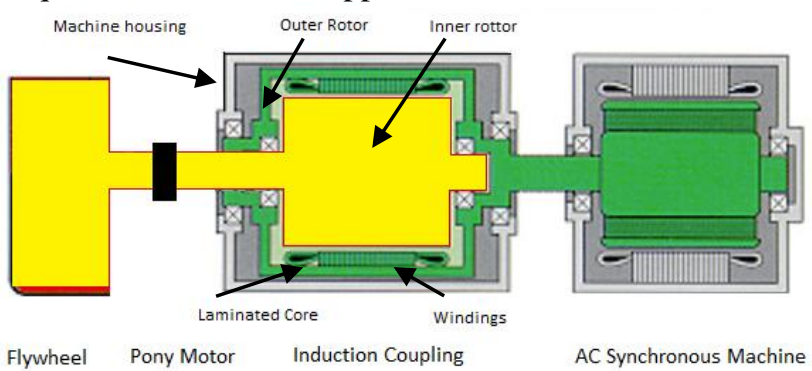

Fig. 1. HITEC RT system

DigSILENT Power Factory is used for simulation of electrical networks. The flywheel frequency behavior is approximated with a model programmed in this environment. It consists of a frame diagram called "composite model" (Fig. 2). The first block represents an AVR. It senses the voltage at the point of connection of the generator. The output signal is the excitation voltage of the synchronous generator. The second block represents a backup generator. The third block controls the induction coupling and the fourth represents the synchronous generator.

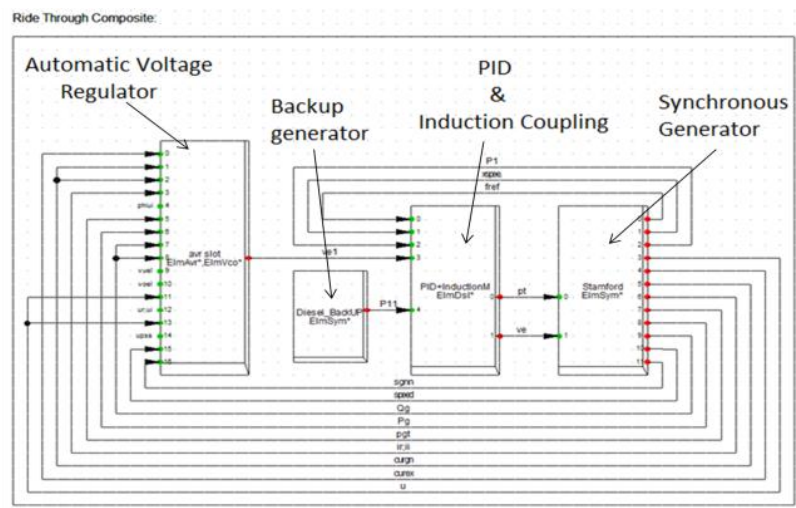

Fig. 2. RT Composite Model.

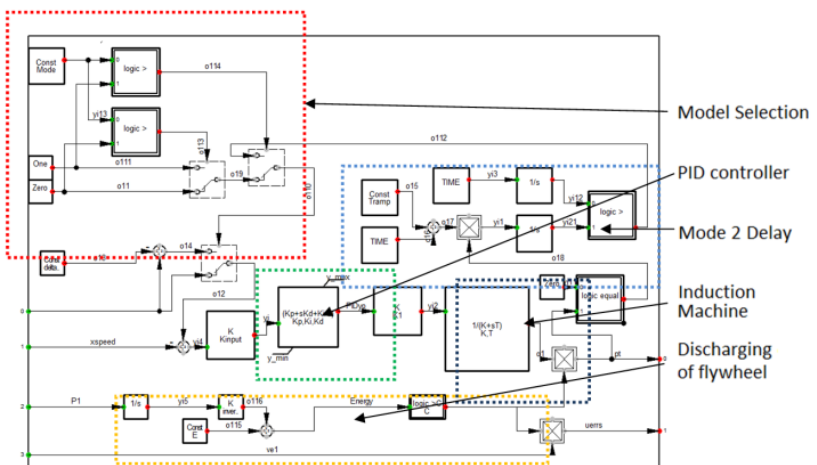

Fig. 3. PID \& Induction coupling block diagram.

The characteristics of the PID \& Induction Coupling element are constructed in a block diagram (Fig. 3). The block diagram has four main parts: The induction machine, a PID controller, a representation of the flywheel's energy, and a delay mode to allow other generators to take over the RT. The first order delay represents the induction machine and has an output signal that is the mechanical torque that will be applied to the synchronous generator.

The induction machine is fed by the DC current controlled by a digital controller (DICON) represented by the PID block. This PID block is limited to have only positive outputs. This is because the DICON cannot accelerate the flywheel and therefore can only discharge it. The flywheel is modeled only as a source of energy. The RT system can provide $1 \mathrm{MW}$ for 15 seconds (it can store up to 15 $\mathrm{MJ})$. The discharging of the flywheel is represented as the integral of the output power of the generator and compared to the stored energy of $15 \mathrm{MJ}$. Once the $15 \mathrm{MJ}$ limit is reached, a zero is multiplied by the output signal of the induction machine and the AVR; the block diagram does not feed the synchronous generator after 15 MJ. Real data and simulation results are compared for validation of the model (Fig. 4). This simulation represents the fundamental behavior of the flywheel in terms of maximum frequency drop and stability time.

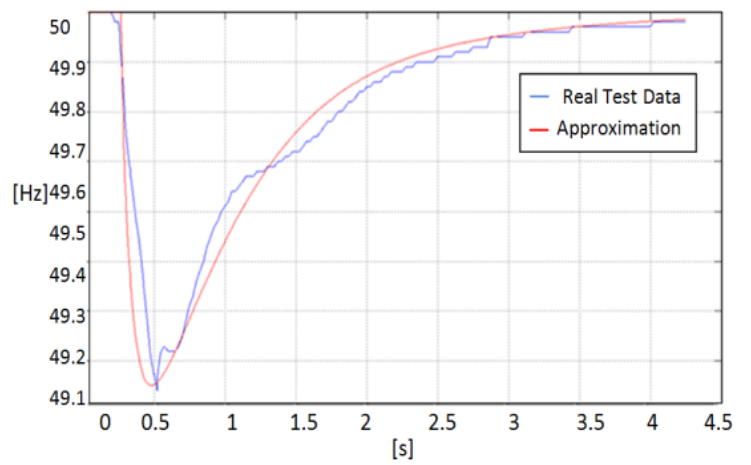

Fig. 4. Validation of the model.

\section{Test network}

\section{A. Network Description}

A network modeled in DigSILENT Power Factory includes three RTs connected in parallel, three CHPs, an industrial load, a city load and a wind farm (Fig. 5). Additionally, the wind farm is represented by eight doubly fed induction generators (DFIG) of $1 \mathrm{MW}$ output power. The grid is providing $1.8 \mathrm{MW}$ to the network at the time of disconnection.

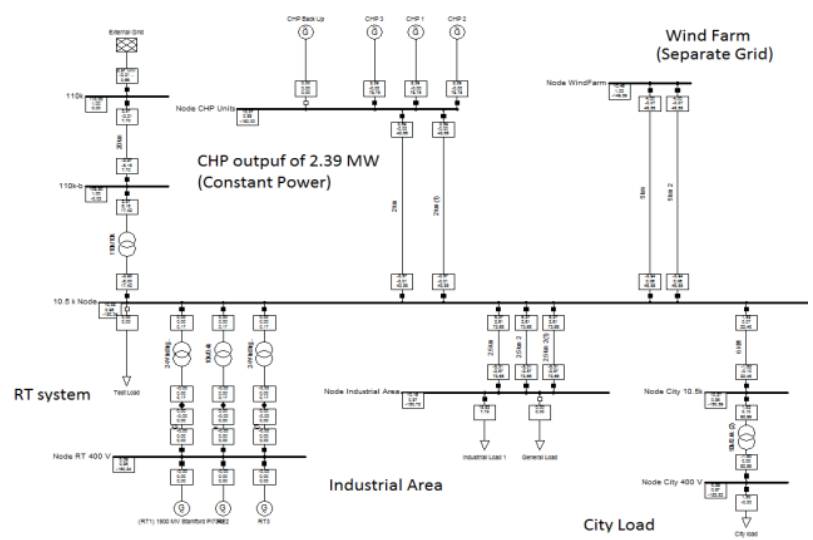

Fig 5. Power Factory Test Network. Grid supplies a MV network. 


\section{B. Scenario 1: Interaction with CHPs}

The scenario describes a city load of $1 \mathrm{MW}$, an industrial load of 5.3 MW, three 2.39 MW CHPs and the wind farm is disconnected. The CHPs have voltage and governor control. The total demanded power from the grid is 1.8 MW. The nominal apparent power of the CHPs is 2.99 MVA. The restrictions for voltage and frequency are based on the Dutch energy regulations $+/-1 \mathrm{~Hz}$ and $+/-10 \%$.

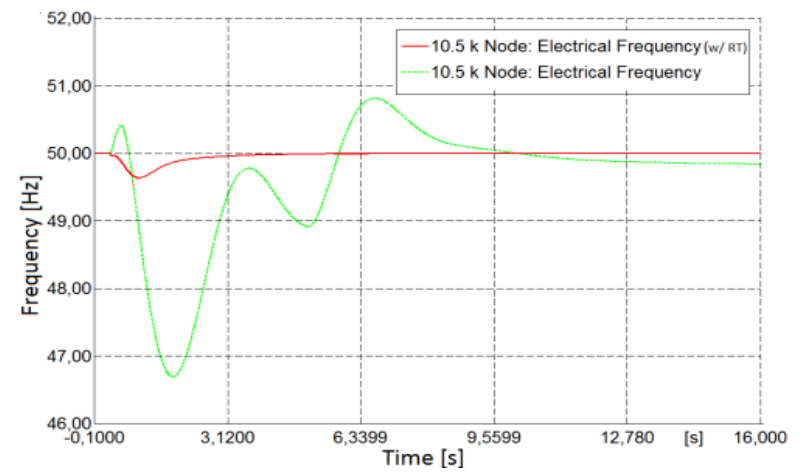

Fig 6. Frequency after disconnection with and without 3 RTs.

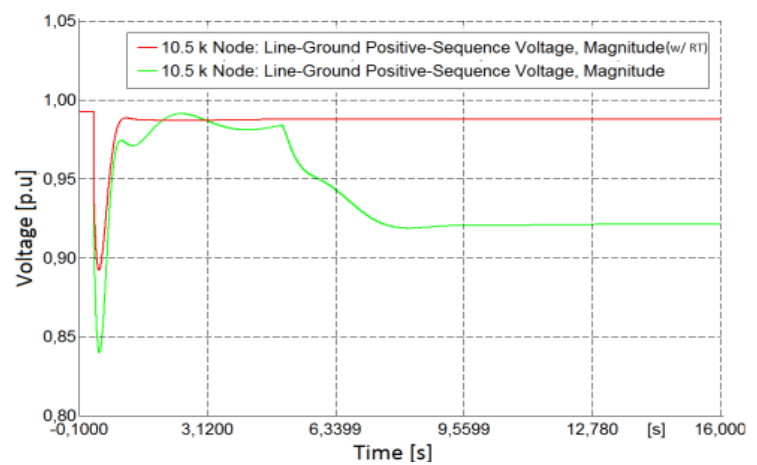

Fig 7. Voltage after disconnection with and without 3 RTs.

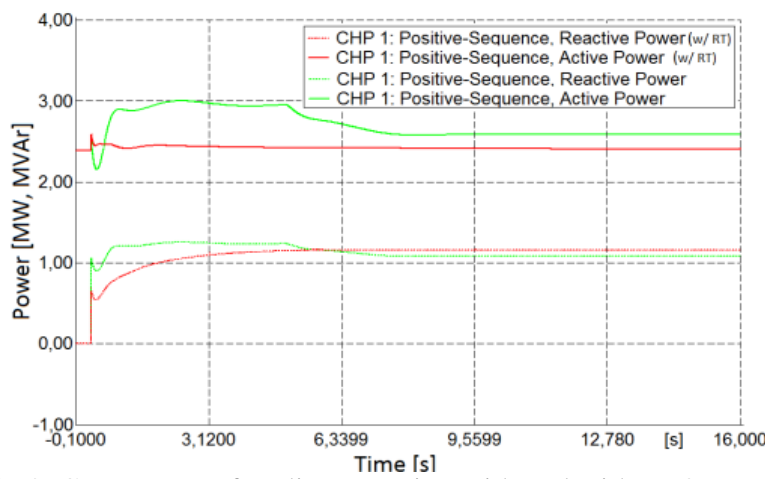

Fig 8. CHP Power after disconnection with and without 3 RTs.

The CHPs increase their power output after disconnection of the grid, however stable operation cannot be achieved without the use of a RT (Fig. 6). Without RT support the frequency's lowest value is $46.7 \mathrm{~Hz}$. Also, the voltage lowest value is $84 \%$ (Fig.7) and the voltage stabilizes around $92 \%$. By using RTs, the frequency drops only to $49.6 \mathrm{~Hz}$ and stabilizes back to $50 \mathrm{~Hz}$. The voltage is also stable with the use of RTs with a fast transient to $90 \%$ after disconnection. The frequency is supported mainly by the RTs while the voltage is mainly supported by the CHPs. This can be seen Fig. 8. Although the RTs also have voltage support this is very limited. The effect is that voltage support is mainly done by other elements in the network. However the number of RTs has an impact on cost and over dimensioning is rarely an alternative. When the use of one RT is used, the frequency drop is within $1 \mathrm{~Hz}$ range (Fig. 9). After the flywheel is depleted, there is another drop in frequency at second 10 which is also within a $1 \mathrm{~Hz}$ range. It appears that the CHPs alone can support the load after the RT runs out of energy (Fig. 9). This is an interesting result because a single RT, providing $1 \mathrm{MW}$, is sufficient to change to autonomous mode and support frequency stability even when the demand from the grid was 1.8 MW.

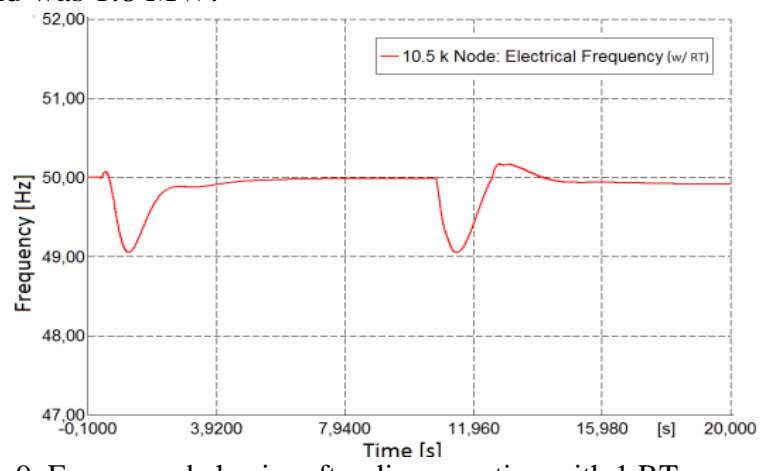

Fig 9. Frequency behavior after disconnection with 1 RT

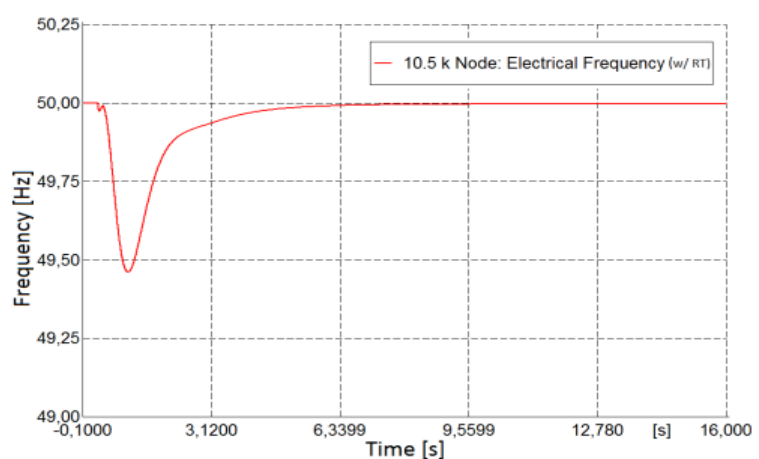

Fig. 10. Frequency behavior after disconnection with $2 \mathrm{RTs}$

However the capacity of the CHPs limits the support of voltage and this one drops to $90 \%$ after the flywheels run out of energy. At this point, the CHPs are overloaded. This means that other reactive power compensation techniques are needed or back-up generators should be started. However, the RT only provides a limited 10 second window to do this. With the support of 2 RTs, the variations in frequency are diminished and the time of support increases from 10 seconds to more than 16 seconds (Fig. 10). After the energy is depleted, the CHPs overload. However, with 16 seconds it is possible to start a backup generator and support the loads.

\section{Scenario 2: CHPs \& wind turbines}

Scenario 2 has a 1.8 MW city load, 15.93 MW and 5.24 MVAr industrial load, three CHPs with 2.39 MW output with governor control and eight $1 \mathrm{MW}$ wind turbines with voltage regulators. The grid supports the network with 2.94 MW and 5.59 MVAr. Fig. 11 shows a frequency drop of 7 $\%$ or $3.5 \mathrm{~Hz}$. The voltage is supported by both the CHPs and the wind turbines. Eventually the CHPs provide all needed reactive power to support the voltage but at the extent of overloading them at second 3 to $122.21 \%$ (Fig.13) and voltage is reduced after 6 seconds (Fig. 12). 


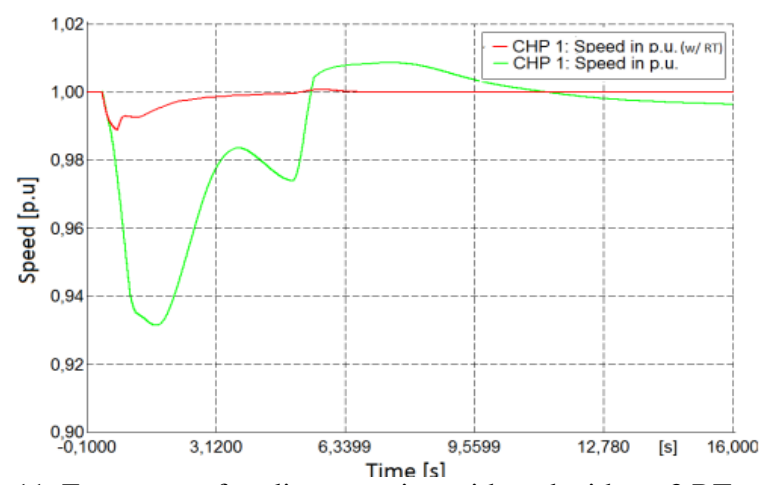

Fig 11. Frequency after disconnection with and without 3 RTs.

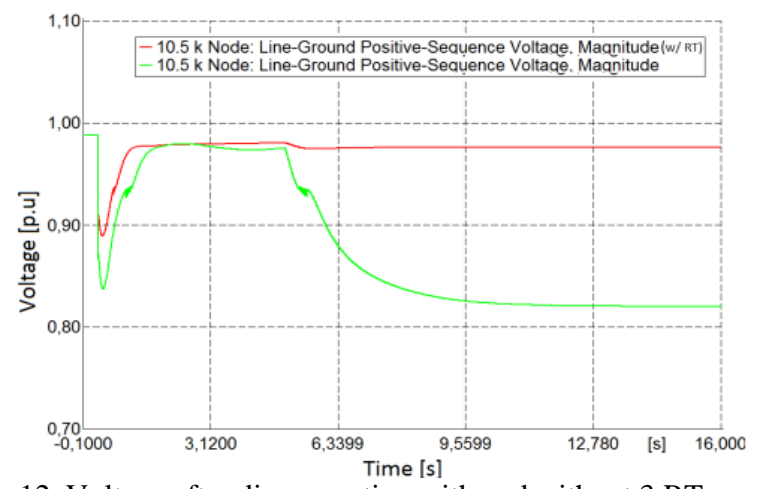

Fig 12. Voltage after disconnection with and without 3 RTs.

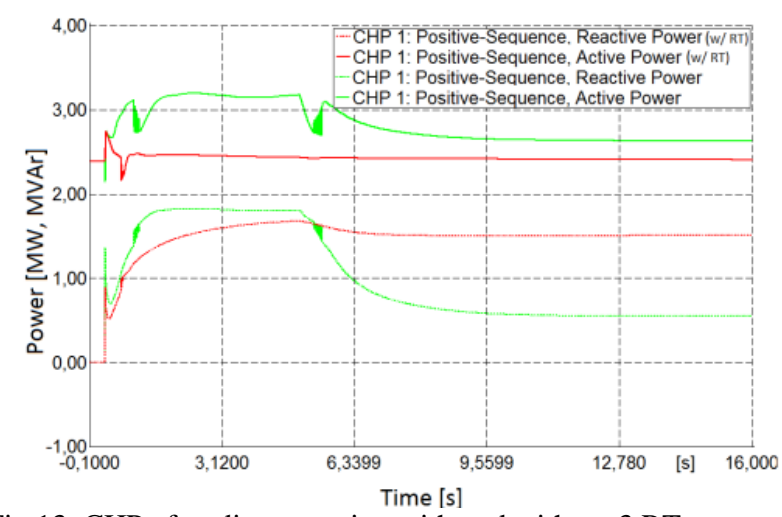

Fig 13. CHP after disconnection with and without 3 RTs.

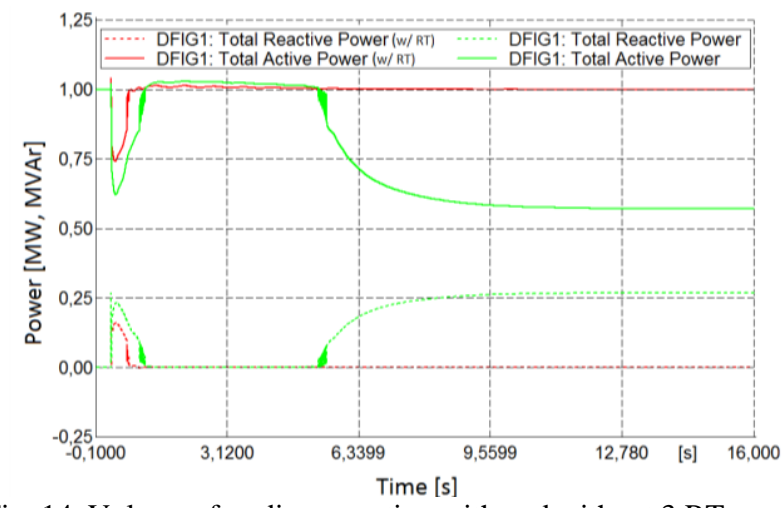

Fig. 14. Voltage after disconnection with and without 3 RTs.

When three RTs are used, the frequency only drops to 99 $\%$ or $0.5 \mathrm{~Hz}$ (Fig.11) and the voltage is supported above 96 $\%$ (Fig. 12). The maximum loading of the CHPs happens at second 5. The overloading will happen only for few seconds and then the loading stabilizes at $94.94 \%$ (Fig. 13). Lowering either the active or reactive power from the values of scenario 2 will avoid overloading of the CHPs.
Therefore, it is important to analyze the amount of reactive power demand when attempting autonomous operation. In Fig. 14 the transient effects on the wind turbines are smaller when RTs are connected and their power output stays constant for as long as the RTs have energy. This figure also shows that the control system sacrifices active energy for voltage support when needed. As a result the CHPs and RTs will have higher demand. The control system needs to be revisited to grant equal demand strain from all the elements in the Microgrid. Nevertheless, in this scenario the RTs can support smooth transition to autonomous and grant sufficient time to start backup generators.

\section{Remote Autonomous Network}

\section{A. Case 1 Network Description}

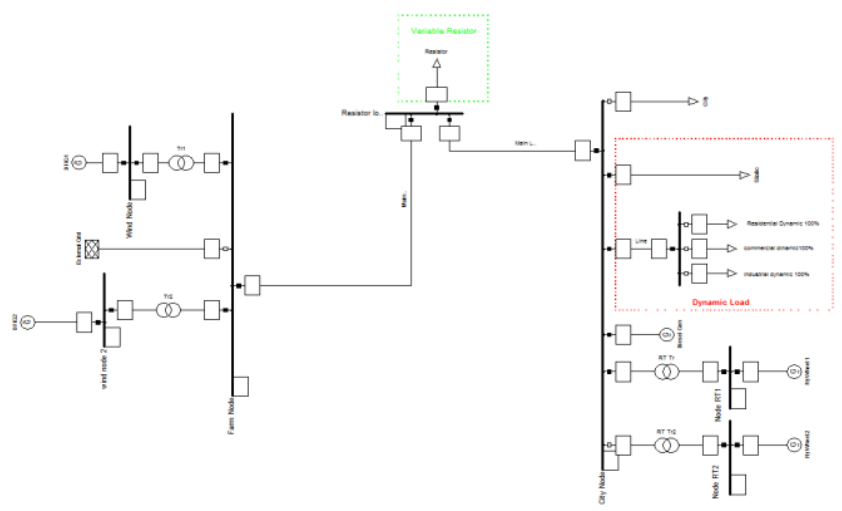

Fig 15. Study Case 1 Power Factory network.

The first study case analyzes an autonomous network which cannot be connected to the grid due to its geographical location. The network has a wind farm (2 $\mathrm{MW}$, a variable resistor, a city load (1.2 MW), and a diesel generator (1.6 MVA). When the wind farm provides $2 \mathrm{MW}$, the variable resistor has maximum value of 1.2 MW, and the diesel generator provides $400 \mathrm{~kW}$. If sudden disconnection of the wind farm occurs (e.g. lightning), the diesel generator increase its power and the variable resistor decrease its load. However, the transition is outside the limits for frequency and voltage stability and the diesel generator could experience pole slipping. The network is built in DigSILENT Power factory (Fig. 15) and the RTs are incorporated.

\section{B. Effect of Dynamic Modeling}

The load nature has effects on the transients of frequency and voltage in networks. For this reason, the loads are simulated using a dynamic model. Loads are defined as commercial, residential and industrial with dependency on voltage and frequency instead of constant impedance [4]. As a result, the behavior and interaction of the loads with other components in the network can be analyzed in more detailed. The AVR of the diesel generator is not considered and no RTs are used. The frequency is affected with bigger magnitude with the use of dynamic modeling and the voltage has higher levels when using dynamic modeling (Fig. 17). This is because the demand for reactive power goes down after disconnection and the demand for active 
power stays high even with large variations of voltage (Fig. $18,19)$.

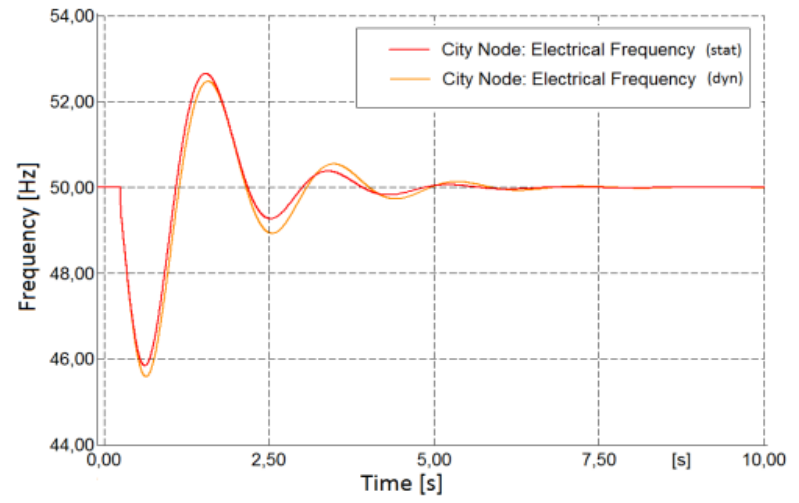

Fig 16. Frequency after disconnection of the wind farm

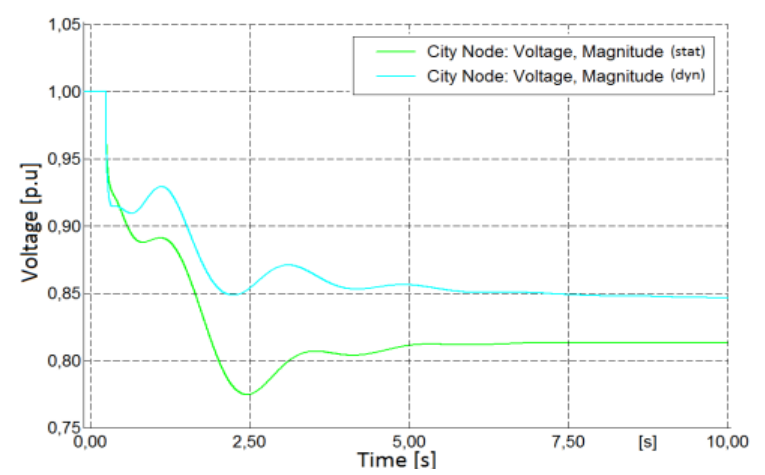

Fig 17. Voltage after disconnection of the wind farm.

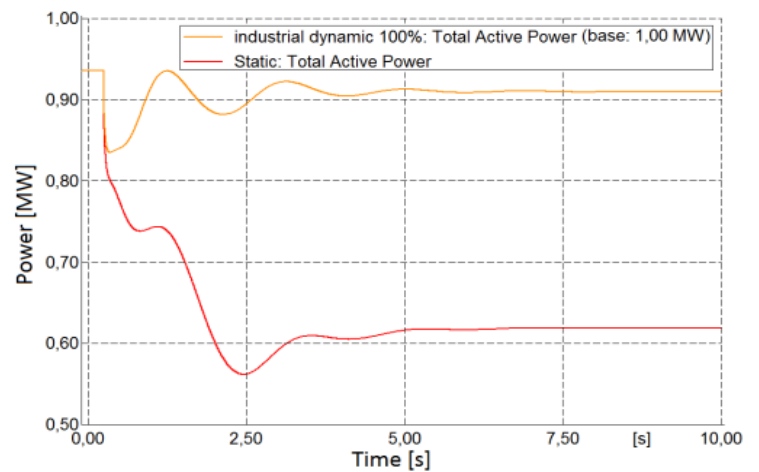

Fig 18. Active Power demand after disconnection of wind farm

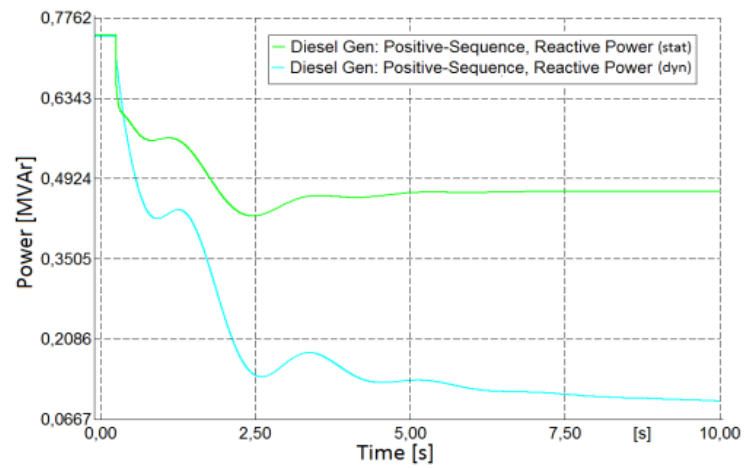

Fig 19. Reactive Power demand after disconnection of wind farm.

\section{Rotary RT Support}

When $1 \mathrm{RT}$ is used the variations in frequency are very small (Fig. 20). The speed of reaction of the RT allows the diesel generator to increase its power output without pole slipping and with little transients in voltage until the diesel generator completely takes over the RT.

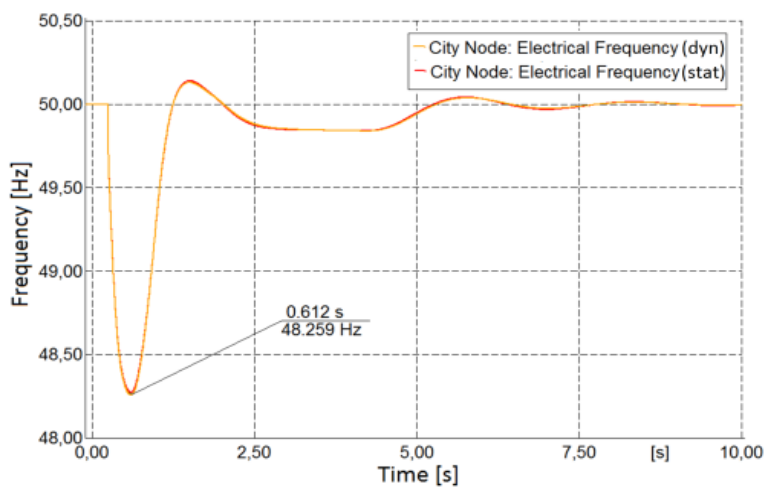

Fig 20. Frequency after disconnection with 1 RT

\section{Tiel Zuid Network}

\section{A. Network Description}

Dispersed generation can increase to the point where the total installed capacity is higher than the load demand. We can see this in a section of the Dutch Electricity grid called "Tiel Zuid" (Fig. 21). It is a medium voltage network at $10.5 \mathrm{kV}$ that consists of 146 synchronous generators, 71 two winding transformers, 574 medium voltage loads and 688 cables with a structure that can be changed from radial one to a meshed one.

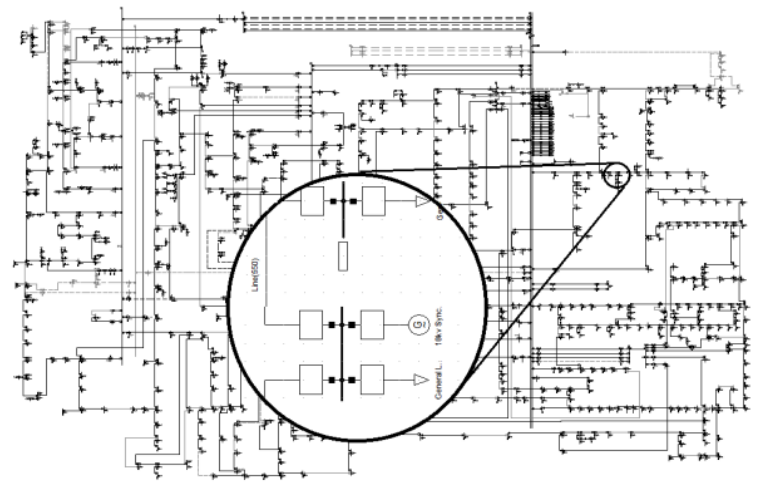

Fig 21. Tiel Zuid Power Factory Network.

During the day, this section of the grid can demand power or export it depending on how many CHPs are working. If disconnection occurs when the grid is exporting power, then transients in voltage and frequency will occur. Simulations with the RT are performed when the grid provides $44 \mathrm{MW}$ of power. At the event of disconnection from the grid, there is lack of power and the CHPs need to increase their output power. In this scenario the variations of frequency and voltage are substantial. Twelve RTs are connected to the main bus bar to withstand the transient behavior of voltage and frequency.

\section{B. Simulation Results}

The Fig. 22 shows that without the use of RTs the voltage transient reaches a minimum value of $47 \mathrm{~Hz}(0.94$ p.u.) and when using RTs the frequency is $48 \mathrm{~Hz}(.96 \mathrm{p} . \mathrm{u})$. Twelve RTs diminished the frequency variations from $47 \mathrm{~Hz}$ to 48 Hz. The RTs will keep giving active power to the network until the flywheels run out of energy. The simulation is extended to see the behavior after 25 seconds (Fig 23). 


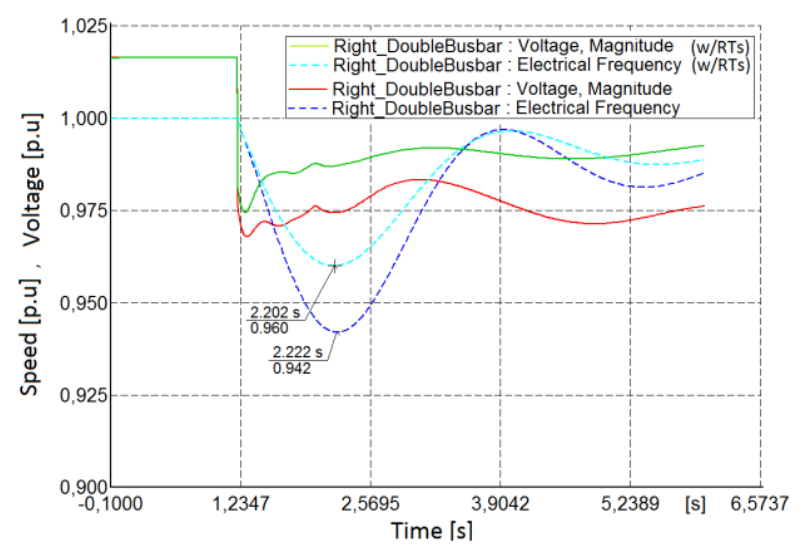

Fig 22. Frequency and Voltage After disconnection without RTs

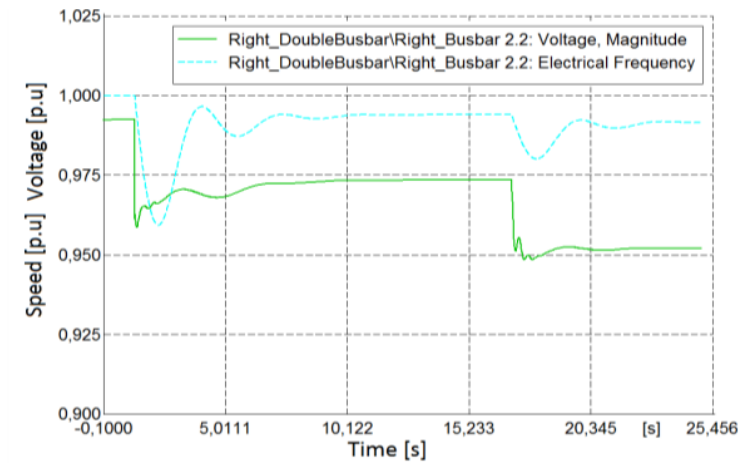

Fig. 23. Frequency and Voltage After depletion of the flywheels.

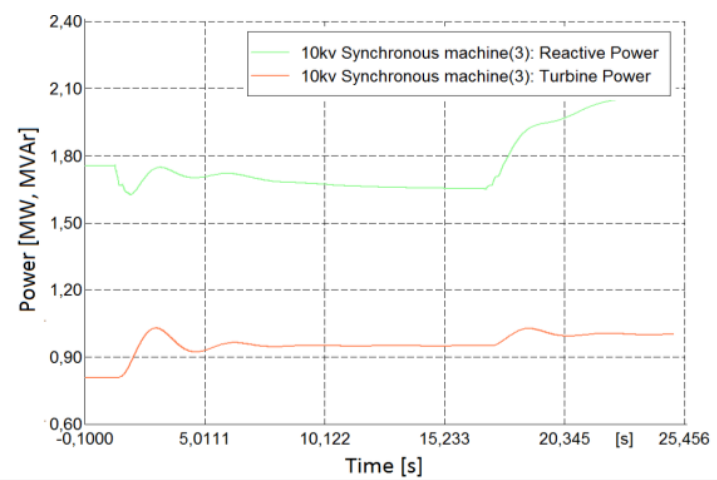

Fig. 24.CHP power output after depletion of the flywheels.

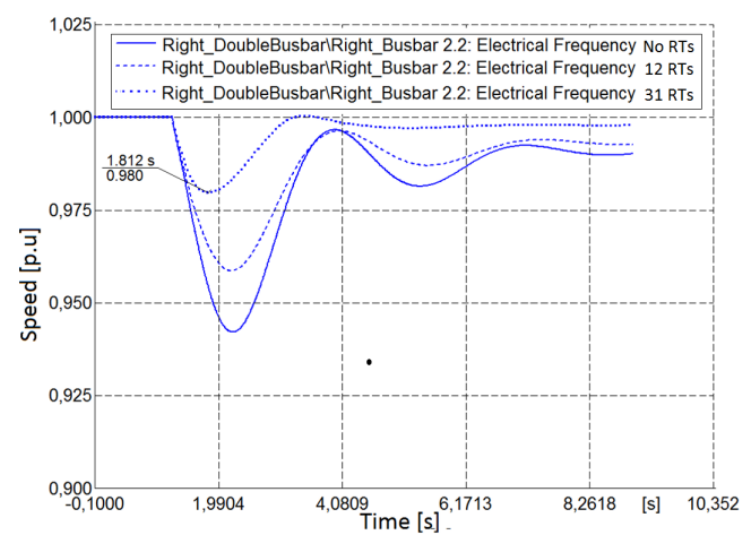

Fig. 25. Frequency and Voltage After disconnection

Even when the lack of energy before disconnection is 44 MW, twelve RTs work for a little more than 15 seconds. This is attributed to the fact that the CHPs increase their active power output as well. This is also the reason why after depletion of the flywheels the variations of frequency are less that $1 \mathrm{~Hz}$. Nevertheless, the CHPs will be overloaded because of the increase in reactive power to support voltage (Fig. 24). Considering that Microgrids may have large penetration of dispersed generation, the number of RTs could be lower because of a combined reaction of the dispersed generation and the RTs thus fewer numbers of RTs per $1 \mathrm{MW}$ of unbalance and the simulations prove this. The number of RTs connected to the Tiel Zuid network can be increased until the transients in frequency are lower than $1 \mathrm{~Hz}$. This happens when there are $31 \mathrm{RTs}$ connected to the network (Fig. 25). In general, the CHPs are expected to support the grid in the long run and RTs should only be used to reach stable operation during disconnection. However, the voltage control and reactive power compensation is still a considerable problem that needs to be addressed with other strategies. Moreover, the number of RTs can be reduced if the governor control is revisited allowing it to respond faster.

\section{Conclusions}

During disconnection or loss of power from renewables, voltage and frequency transients need to be addressed to secure stable performance of the network. Several cases were simulated using DigSILENT Power Factory with a RT model. The simulations suggest that for maximum change in power output, a FRT supports the load while other generators increase their power output or to backup generators and stand-by CHPs to start. Any other variation in power supply can be successfully supported by the RT's fast injection of power. The results also indicate that the transient behavior of voltage and frequency deeply depend on the control strategy used for each of the elements of the network. The type of governors and AVR affects the behavior of the transients. The available DigSILENT Power Factory basic models need to be adapted to match the configuration of commercial AVR and governors. Moreover, in future autonomous grids, the dispersed generation control system needs to take into account the interacting elements, including storage systems like the flywheels. An intelligent structure needs to be designed to sense multiple elements of the Microgrid on a higher level and control the reaction of stand-by and active generators to different events.

\section{References}

[1] Badrul H. Chowdhury Nikhil K. Ardeshna, "Supporting Islanded Microgrid Operations in the Presence of intermittent Wind Generation," pp. 1-8, 2010.

[2] Briank K, Johnson, Mariesa L. Crow, Aysen Arsoy, Yilu Liu Paurlo F. Ribeiro, "Energy Storage Systems for Advanced Power Applications," Proceedings of the IEEE, vol. 82, no. 12, pp. 1744-1756, December 2001.

[3] N.Hatziargyriou, J. Mutale, P.Djapic, N.Jenkins J.A Pecas Lopes, "Integrating distributed generation into electric power systems: A review of drivers, challenges and opportunities.," 2006.

[4] J.Jatskevich T. Aboul-Seoud, "Dynamic Modeling of Induction Motor Loads for Transient Voltage Stability Studies," 2008 IEEE Electrical Power \& Energy Conference, 2008 